Recibido: 22/01/2018 --- Aceptado: 07/03/2018 --- Publicado: 15/07/2018

\title{
ESTRATEGIAS PARA LA FORMACIÓN PROFESIONAL EN PERIODISMO RADIOFÓNICO: EXPERIENCIAS Y PROPUESTAS \\ STRATEGIES FOR PROFESSIONAL TRAINING IN RADIO JOURNALISM: EXPERIENCES AND PROPOSALS
}

Zenaida Costales Pérez ${ }^{1}$ : Universidad de La Habana. Cuba

costaleszenaida@gmail.com

C. Ana Teresa Badía Valdés: Universidad de La Habana. Cuba abadia@fcom.uh.cu

\section{RESUMEN}

Esta propuesta recoge algunas bases para la formación profesional de la asignatura de Periodismo Radiofónico en la Facultad de Comunicación de la Universidad de La Habana. El texto explora en la construcción simbólica para el escenario cubano en los contextos culturales contemporáneos, sobre el eje transversal de la enseñanza radial. Interpretar la realidad y comunicarla, así como la combinación de la teoría y con la práctica es parte de las esencias pedagógicas que contribuyen a entender la complejidad de nuestros días. En ese escenario el profesor debe asumir un liderazgo noticioso comunicativo y pedagógico que deberá influenciar en la formación profesional.

PALABRAS CLAVES: radio; enseñanza; teoría; práctica; pedagogía.

\section{ABSTRACT}

This proposal gathers some bases for the professional formation of the subject of Radiophonic Journalism in the Faculty of Communication of the University of Havana. The text explores the symbolic construction for the Cuban scenario in contemporary cultural contexts, on the transversal axis of radio education. Interpreting reality and communicating it, as well as the combination of theory and practice, is part of the pedagogical essences that contribute to understanding the complexity of our days. In this scenario, the teacher must assume a communicative and pedagogical news leadership that should influence professional training.

KEYWORDS: radio; teaching; theory; practice; pedagogy.

\footnotetext{
${ }^{1}$ Zenaida Costales Pérez. Universidad de La Habana. Cuba costaleszenaida@gmail.com
} 


\section{ESTRATÉGIAS PARA A FORMAÇÃO PROFISSIONAL EM JORNALISMO RADIOFÔNICO: EXPERIÊNCIAS E PROPOSTAS}

\section{RESUME}

Esta proposta percorre algumas bases para a formação profissional da matéria de Jornalismo Radiofônico na Faculdade de Comunicação da Universidade de Habana. 0 texto explora na construção simbólica para o cenário cubano nos contextos culturais contemporâneos, sobre o eixo transversal do ensinamento radial. Interpretar a realidade e comunica-la, assim como a combinação da teoria e com a pratica é parte das essências pedagógicas que contribuem a entender a complexidade de nossos dias. Neste cenário o professor deve assumir uma liderança noticiosa comunicativa e pedagógica que deverá influenciar na formação profissional.

PALAVRAS CHAVE: Rádio - Ensinamento - Teoria - Prática - Pedagogia.

\section{Cómo citar el artículo}

Costales Pérez, Z.; Badía Valdés, A. T. (2018). Estrategias para la formación profesional en periodismo radiofónico: experiencias y propuestas. [Strategies for professional training in radio journalism: experiences and proposals]. Revista de Comunicación de la SEECI, 46, 53-63. doi: http://doi.org/10.15198/seeci.2018.46.53-63. Recuperado de http://www.seeci.net/revista/index.php/seeci/article/view/508

\section{INTRODUCCIÓN}

En los países en desarrollo, al menos $75 \%$ de los hogares tienen acceso a la radio. (UNESCO, 2012). Esa cifra concede importancia al medio.

En Cuba, la radio al escuchar a su audiencia y responder sus necesidades, provee tanto aristas como voces para abordar los desafíos en la actualidad. (Rebelde, 2018). Su enseñanza abarca en primera instancia una dimensión pedagógica anclada a la responsabilidad del futuro profesional, su ética, su ideología profesional, y la necesidad de considerar las estéticas de las nuevas narrativas en el momento de ejecutar los ejercicios prácticos en el aula.

Se propone la enseñanza de la asignatura Periodismo Radiofónico en la Facultad de Comunicación de la Universidad de La Habana como una reflexión experiencial, alterna y simultánea que involucra la producción periodística radiofónica y la docencia universitaria. Se pusieron en práctica además herramientas educativas estrechamente relacionadas con la interpretación del contexto político y sociocultural. Como expresa el investigador colombiano Jesús Martín-Barbero (2010), el primer desafío de la comunicación hoy en la sociedad del conocimiento es poner a dialogar la universidad con su sociedad.

La potenciación del relato radiofónico, su construcción dramatúrgica, que incluye el papel del docente en la concepción de la clase, así como la innovación individual y 
colectiva para enfrentar la transformación social, se convierten en modos y medios experimentales de la experiencia pedagógica.

\section{OBJETIVOS}

Como objetivos de la investigación se plantean: reflexionar acerca de rutas para la búsqueda de una formación estrechamente relacionada con la experiencia docente radiofónica interactiva y que transitan por el aprendizaje teórico, la experiencia práctica, el aporte colectivo e individual a un determinado proyecto, la evaluación conjunta de los resultados y la interpretación crítica del contexto. También mostrar los aportes, que desde experiencias teórico-práctica en el ámbito universitario, contribuyan a la búsqueda de un modelo de formación novedoso en tanto asumen el acervo del pensamiento crítico. Se incluye además evidenciar las potencialidades que la enseñanza de la radio ofrecen para construir escenarios a través de las historias de vida que a su vez se conviertan en espacios estratégicos de la construcción simbólica contemporánea cubana desde prácticas de reconocimiento y auto-reconocimiento que pudieran ser útiles en otros campos de la formación docente. Y por último, diseñar una propuesta desde el punto de vista teórico-metodológico para la contribución al mejoramiento de la enseñanza de la asignatura de Periodismo Radiofónico en la universidad.

\section{METODOLOGÍA}

Este estudio se sustenta epistemológicamente sobre el paradigma interpretativo de la investigación que busca comprender, describir e interpretar la realidad relacionada con el objeto de estudio. Metodológicamente se utiliza un diseño cualitativo al describir e interpretar los fenómenos de la realidad. Contempla la realización de un ejercicio innovador y experimental (Galán y Quintanal, 2012). Posteriormente, y sobre la base de los resultados de ese ejercicio inicial se proponen algunas bases teóricas y metodológicas para la enseñanza de la asignatura. Se asume el procedimiento inductivo, lo que permite establecer una relación estrecha con el objeto con la finalidad de poder penetrar en su esencia, en tanto resulta necesario establecer las regularidades que posibilitan la elaboración de cambios en la propuesta de enseñanza. Se emplean métodos como el análisis histórico-lógico de la literatura pedagógica; la generalización teórica para la interpretación de la información obtenida; el análisis-síntesis y la inducción-deducción; el análisis documental; y la triangulación de datos.

La población y muestra están en la Facultad de Comunicación de la Universidad de La Habana donde se forman profesionales de tres carreras, de ciencias información, comunicativas y periodísticas. En la carrera de Periodismo se imparte la asignatura de Periodismo Radiofónico. Este trabajo de investigación se realizó con un grupo de 46 estudiantes en el curso académico 2014-2015. Sobre la base de ese diagnóstico, se establece la propuesta teórico-metodológica para la enseñanza de la asignatura. 
Costales Pérez, Z.; Badía Valdés, A. T. Estrategias para la formación profesional en

periodismo radiofónico: experiencias y propuestas

\section{DISCUSIÓN}

\subsection{Pedagogía Radiofónica}

Desde la universidad, la educación radiofónica, debe modificarse al ritmo de la sociedad y estar a tono con el cambio de los modelos tanto educativos como comunicativos que permitan preparar a estudiantes y profesores para los desafíos del presente y el futuro.

Nadie duda de la existencia de un medio de comunicación reconocible empíricamente tanto por sus características tecnológicas como por los determinados modos generalizados de operarlo. Las distintas prácticas profesionales que se forman alrededor de él y los conocimientos académicos que se desarrollen dan lugar al reconocimiento de disímiles modos de pensar la radio, que obviamente no son casuales sino que son resultado de múltiples concepciones acerca de la comunicación y de los medios de comunicación. (Canavilhas, 2011).

Para asumir entonces la pedagogía de la comunicación radiofónica, frente a un estudiante que siempre demanda una mejor propuesta didáctica, el docente debe saber que el alumno se apasiona más por el hacer, y hasta a veces aceptando que para producir radio no se necesita aprender el fundamento de la comunicación radiofónica. De manera más categórica lo plantea el mismo Jesús Martín Barbero (2005) cuando afirma: "el problema ya no es la ponderación del peso que en la formación del comunicador deben tener los distintos saberes y destrezas sino qué tipo de reflexión teórica puede articularse al 'hacer comunicación' sin quedar absorbida o neutralizada por la razón tecnológica y la 'expansión' de la lógica mercantil a modelo de sociedad". (p.124).

Esa subvaloración hacia la formación teórica se incrementa en la medida en que las diversas metodologías empleadas para la Pedagogía de la comunicación radiofónica, se agotan en modelos y ayudas tradicionales ya gastadas en su aplicación, que no logran cautivar al estudiante de la misma forma que le seduce la posibilidad de estar en una cabina de radio o en un estudio con los componentes tecnológicos de estos tiempos.

Mata (1998) considera que "la posibilidad de restituirle (a la radio) su unidad como objeto completo y complejo en torno al cual el pensar y el hacer no sean categorías y momentos disociados, antagónicos, o simplemente vinculados por el nexo de la aplicabilidad de unas ciertas teorías en la acción, sino momentos diferentes, pero convergentes" (p.94).

Aunque la intención del educador de radio sea formar con un amplio criterio que integre diversas perspectivas del medio, el proceso pedagógico de la comunicación radiofónica se ve perturbado, en muchas ocasiones, por esta predilección hacia prácticas de carácter más empírico y técnico, al menos, en la percepción inicial del estudiante.

La defensa académica en torno a los fundamentos teóricos, enriquece y sustenta la producción de una comunicación radiofónica con contexto y profundidad conceptual. Pero, ¿qué factores comienzan a desempeñarse en la tarea de la enseñanza de la radio para que se preste atención tanto a lo teórico como a lo práctico? 


\section{Costales Pérez, Z.; Badía Valdés, A. T. Estrategias para la formación profesional en}

periodismo radiofónico: experiencias y propuestas

La enseñanza del periodismo radiofónico se convierte en un ejercicio pedagógico trascendental como herramienta del discurso contemporáneo por excelencia: seducción, espectáculo, imaginario lúdico, posibilidades de apropiación y construcción de discursos. Ello unido al desarrollo y expansión de las Tecnologías de la Información y las Comunicaciones (TICs). En la combinación de los anteriores elementos radica uno de los caminos pedagógicos para la articulación de una teoría-práctica transformadora, involucrada directamente con el contexto circundante.

La potenciación del relato radiofónico, su construcción dramatúrgica (que incluye el papel del docente en la concepción y puesta en escena de la clase) y la innovación individual y colectiva para encarar la transformación social, se convierten en modos y medios experimentales esenciales de la experiencia pedagógica.

La incidencia del mágico relato radiofónico introduce nuevas formas expresivas, estéticas, y la posibilidad de comunicar sentidos profundos, que reflejan realidades múltiples y diversidades culturales. Deben emplearse variados lenguajes, así como nuevas formas de expresión, de interacción, de creatividad y de imaginación.

Con narrativas musicales, sonoras y auditivas se pueden dibujar y manifestar nuevas formas de pensar y de relacionarse. La variedad y la multiplicidad de interpretaciones sonoras contribuyen a descubrir otro mundo dentro del mundo y a los /as otros /as en toda su dimensión. De esta manera, y no por gusto, la periodista chilena Perla Wilson propone un cruce posible: "que el relato radiofónico sea una memoria".

En el proceso de la enseñanza/aprendizaje de la comunicación radiofónica hay otro elemento significativo que apunta a formar: la especificidad del discurso del medio que le es propio y que salvaguarda muchas de las características que lo hacen diferente de otros. Como arte, la radio tiene una preocupación estética muy legítima: su relación con el oyente se deriva de la seducción y de lo apropiado de sus contenidos. (Morley, 1996). Aspectos como variedad, calidad sonora, ilación del relato, apropiación de los cuatro elementos del discurso radiofónico (la palabra, la música, los efectos sonoros y el silencio) son, de algún modo, garantía de una propuesta estética. "La radio no puede prescindir del arte sin riesgo de que su mensaje, aun cuando pueda revestir algún grado de utilidad, resulte una machacona letanía incapaz de gratificar, de provocar la fruición estética". (Haye, 1998, p.2)

Por todo ello creemos que el docente debe también hacer arte con su clase: es un actor que mediatiza el conocimiento y la experiencia del educando. Es al mismo tiempo, un facilitador del encuentro del estudiante con su entorno. Si un docente no maneja las implicaciones políticas y éticas de su época, no tendrá manera de inducir esa lectura sobre la realidad a sus estudiantes con dramaturgia y espectacularidad como sugiere el periodismo contemporáneo. Utilizar las posibilidades lúdicas del aprender creativo que un medio de comunicación permite, en particular la radio, por su posibilidad de combinar el hacer con el pensar nos conduce por las rutas de una suerte de dramaturgia pedagógica.

"La educación es una obra de arte"..."en el sentido que el educador también es un artista: él rehace el mundo, él redibuja el mundo, repinta el mundo, recanta el mundo, redanza el mundo." (Freire, 1992, p. 77). 
Por ello llegar a la clase, implica envolver la atmósfera creativa y mágica radial del aula con informaciones, noticias y productos comunicativos que reflejen la realidad. La del momento de la clase: La inmediatez de la radio debe también hacer gala en la clase de periodismo radial. (Cebrián, 2004).

Tal como lo explica Lalinde (1992) "La noticia es una institución social y una realidad histórica, legitimada socialmente para cumplir la función de estructurar la realidad misma". (p.5).Es una creatividad en la cotidianeidad, en el día a día, hora a hora, minuto a minuto de la secuencialidad para la comunicación inmediata con la audiencia. $Y$ ello puede funcionar en el aula como una especie de dispositivo lúdico que discurre en sensaciones únicas. Mística que reúne a cuerpos grupales y cuerpos individuales que escuchan decir, resuenan, modelan, reescriben y resignifican en su avidez por continuar tejiendo la trama, creando retóricas ficcionales, de expresividades y de estéticas narrativas.

\section{2 Las clases espacio físico o emocional}

Como parte de la experiencia, se convirtió el espacio físico del aula en un escenario de reconocimiento y auto-reconocimiento, con un carácter altamente inclusivo, que permitió potenciar el espíritu movilizador-transformador de los alumnos.

"Si comunicar es compartir la significación, participar es compartir la acción. La educación sería entonces el decisivo lugar de entrecruce. Pero para ello deberá convertirse en el espacio de la conversación de los saberes y las narrativas que configuran las oralidades, las literalidades y las visualidades. Pues desde los mestizajes que entre ellas se traman es donde se vislumbra y expresa, toma forma el futuro."(Barbero, 2002, p.1)

Como parte de la estrategia pedagógica se organizaron visitas y se estructuraron las clases de manera auténticamente mediáticas en diferentes emisoras radiofónicas. Ello cambió la óptica del estudiante, ya que le permitió constatar la agilidad y el dominio que exige el medio. Para los estudiantes la clase en cabina, en el estudio de radio, se hizo más atractiva, pues es ahí donde pudieron constatar sus fortalezas. Para los alumnos, estar en la cabina fue estar en la práctica y la práctica constituye, aparentemente, la forma más tangible de "hacer radio". "El educador necesita del educando así como el educando necesita del educador, ambos se educan, aunque las tareas de ambos sean específicas (...)"(Freire, 1992, p. 77).

Otro aspecto relevante fue la socialización de productos radiales, de materiales teóricos y de las producciones científicas de los estudiantes, como táctica pedagógica que permitió que tanto los alumnos como los docentes clarificaran conceptos y aprendieran nuevas formas de trabajo de la comunicación radiofónica. En este sentido resalta además, la necesidad la búsqueda de vínculos fuertes con proyectos reales fruto de su interacción durante su práctica laboral. En nuestra experiencia pedagógica, los alumnos colaboran en emisoras nacionales, provinciales y municipales, espacios donde han de articular el conocimiento teórico con la praxis. 
El estudio del periodismo radiofónico se articuló con materias optativas como: Locución Informativa, Construcción del Relato Audiovisual, Sonido y Relato Audiovisual, Edición Digital, que posibilitaron que, la asignatura de Periodismo Radiofónico se convirtiera junto a la Práctica Laboral en disciplina integradora para que los alumnos se familiaricen más con la actividad profesional mediática, la vida cotidiana, las ideologías profesionales y rutinas productivas de los periodistas radiales cubanos.

Para obtener además una mayor efectividad en el aprendizaje, se utilizaron estrategias analíticas como el desmontaje de productos comunicativos propios del medio, como vía de llegar a la producción de materiales de manera más consciente y crítica.

Durante la investigación se confirmó la necesidad de una estrategia de formación docente capaz de preparar profesionales que se inserten activamente en el mundo contemporáneo y en el entorno sociocultural cubano lo cual conlleva una concepción del periodista como un profesional que además de comunicar la realidad a través de la prensa escrita, radial, televisiva y digital, tenga la responsabilidad de pensarla.

La presencia en el aula de reconocidas personalidades de la radio, quienes juntos compartan experiencias y clases, también ofreció una fortaleza pedagógica irrefutable. El profesor uruguayo Mario Kaplún (1997) usaba expresiones como: "se aprende al comunicar", "conocer es comunicar" o "del educando oyente al educando hablante", y afirmaba: "educarse es involucrarse y participar en un proceso de múltiples interacciones comunicativas". (p.2).

Como parte de la investigación se incluyó la construcción de auto relatos de los estudiantes desde las perspectivas de un discurso radiofónico innovador. Consistió en que los alumnos conformaron estrategias de formación que apuntaran al auto reconocimiento y a la inserción en los contextos. En ellos se articulaban vida personal y entorno social. La idea fue una suerte de lienzo sonoro donde estuvo presente el relato las historias de vida con el uso de los elementos del discurso radiofónico. El objetivo obedecía fundamentalmente a aprender a escucharse y compartir con otros su propia construcción de memoria sonora sobre una época y realidad común para todos: Sus vidas, un país. Trabajaron desde la intención de incorporar al relato no solo la música, sino también efectos sonoros, la palabra, el silencio. La mayoría de los realizadores de historias (los propios alumnos) optaron por no utilizar la figura clásica de un relator 0 locutor que contara la historia. Los mismos protagonistas adquieren significación central como punto de partida, hilo conductor de la mayoría de los trabajos. En los relatos estuvo presente la temática del trabajo no estatal y su papel protagónico en el movimiento diario citadino, el que pregona con nuevas tecnologías sus ricos tamales o dulces por el eterno reto de la supervivencia.

Los relatos radiofónicos articulados por los estudiantes contaran su país desde los sonidos. Ello arrojó una recopilación de sonoridades que identificaban acontecimientos ligados a la historia cubana desde una perspectiva generacional e innovadora. Fue un ejercicio de autodescubrimiento e impacto para alumnos y profesores; requirieron de la apropiación de los elementos discursivos radiofónicos con recursos sencillos y disponibles, capacidad creadora y sostenibilidad en su aplicación a lo largo del tiempo. 
Esa mirada exige, como describe Mata (2012) de "una relación comunicativa como espacio de práctica significante, de producción de sentido; de interacción, de diálogo; una experiencia cultural inserta en una trama textual y contextual (discursiva e histórica) que la constituye a su vez y la modela". (p.6).

\subsection{Una propuesta para la enseñanza de la asignatura Periodismo Radiofónico}

La realización del ejercicio experimental y la triangulación de datos obtenidos desde el análisis bibliográfico y documental, posibilitan la articulación de varios elementos en una propuesta teórico-metodológica para la enseñanza del periodismo radiofónico en la Facultad de Comunicación de la Universidad de La Habana que incluye:

a) Concepción sistémica de la asignatura.

Cuando se enseña radio, debe ahondarse en los ejes de comunicación relativa lineal entre receptores y emisores. En sus reflexiones sobre el aprendizaje como clave de la educomunicación, Prieto (2006, p.7) apunta: «Es muy difícil aprender de alguien con quien poco me comunico, mal me comunico o no me comunico; es muy difícil aprender de alguien con quien no comparto tiempos, porque ni él ni yo los tenemos; es muy difícil aprender de alguien en quien no creo; es muy difícil enseñar, promover y acompañar el aprendizaje de las jóvenes y los jóvenes estudiantes si ha sido minada mi voluntad de aprender».

La existencia y consolidación de asignaturas de la malla curricular de la carrera de Periodismo como: (Locución Informativa, Construcción del Relato Audiovisual, Sonido y Relato Audiovisual, Edición Digital) contribuyen hoy a que la enseñanza radiofónica sea una auténtica disciplina integradora junto a la Práctica Laboral, todas orientadas a la adquisición de habilidades cognoscitivas, metodológicas, tecnológicas y lingüísticas, así como al desarrollo de competencias interpersonales, referidas al cultivo de capacidades individuales y sociales. Esas competencias llegan a ser espacios de integración y destrezas que deben ser adquiridas por los estudiantes a lo largo de su proceso formativo.

b) Articulación de la enseñanza de la asignatura con el contexto.

El hecho de observar, criticar y explicar la realidad debe ser hoy, más que nunca, una labor del futuro periodista que no puede verse como un simple narrador de la actualidad, sino que debe apelar a su profesionalidad y a su buen hacer e intentar interpretar la realidad para exponerla críticamente. Dicha realidad se encuentra mediada por características sociales, económicas, culturales y políticas, entre otras. La introducción de ejercicios y clases relacionadas con el contexto posibilita el desarrollo de la capacidad creativa, crítica e interpretativa, en estrecha relación con la estética radiofónica. 
c) Vinculación de la clase con la actualidad noticiosa y mediática.

La introducción de la actualidad noticiosa como parte del contenido de la clase conlleva a la renovación de la enseñanza radiofónica. Para su debate se debe aplicar el uso de las técnicas del relato radiofónico, así como su construcción dramatúrgica, de manera que refleje dicha actualidad.

d) Vinculación de la teoría y la práctica.

Para enseñar a hacer radio desde la universidad ha de mantenerse el contacto con la práctica de producción en los medios. Pueden ser concebidas al finalizar cada semestre, así como de manera sistemática, de forma que los estudiantes apliquen las teorías obtenidas como parte del proceso enseñanza-aprendizaje.

También la introducción de ejercicios y clases prácticas posibilitó el desarrollo de la capacidad creativa, crítica e interpretativa, en estrecha relación con la estética radiofónica.

e) Consideración del influjo de las nuevas tecnologías.

Las nuevas tecnologías transforman tanto el proceso de producción tradicional de los medios como las maneras de consumo. Por tanto, la formación de los nuevos periodistas radiales $y$, en general de los profesionales de la comunicación, debe dar cuenta del uso de las nuevas herramientas tecnológicas. Como parte de la enseñanza de la asignatura, se deben introducir conferencias sobre el desarrollo de competencias en TICs, el manejo de softwares, y las nuevas características del proceso de comunicación a partir de la consolidación de esas tecnologías.

\section{CONCLUSIONES}

La educación radiofónica universitaria, debe modificarse al ritmo de la sociedad y estar a tono con el cambio de los modelos tanto educativos como comunicativos que permitan preparar a estudiantes y profesores para los desafíos comunicacionales del presente y el futuro.

Los estudiantes deben tener presente que para la trasformación necesaria de la comunicación radiofónica cubana se necesita de un mayor protagonismo del ciudadano común.

Las aulas son auténticos espacios estratégicos simbólicos. Hoy los saberes que enseñamos en nuestras universidades, están atravesados además por saberes tecnocomunicativos. Un periodista radial en formación debe desarrollar competencias comunicativas para reelaborar y transmitir pensamiento científico social y comunicacionalmente relevante a la coyuntura que vive su país. En ese mismo sentido, es importante que los alumnos conozcan el discurso propio de la radio. 
Costales Pérez, Z.; Badía Valdés, A. T. Estrategias para la formación profesional en periodismo radiofónico: experiencias y propuestas

Luego de triangular los resultados de las experiencias del ejercicio innovador, desde esta investigación, es posible sugerir algunos elementos teóricos y metodológicos que contribuirán al mejoramiento de la enseñanza de la asignatura de Periodismo Radiofónico: la concepción sistémica de la asignatura; la articulación de la enseñanza de la asignatura con el contexto; la vinculación de la clase con la actualidad noticiosa y mediática; la vinculación de la teoría y la práctica; y la consideración del influjo de las nuevas tecnologías.

\section{REFERENCIAS}

Alfaro, Rosa M. (1998). Comunicación Educación: una alianza estratégica de los nuevos tiempos. Santa Fe de Bogotá.

Canavilhas, J. (2011). El nuevo ecosistema mediático. Index. comunicación, 1, 13-24. Recuperado de http://el nuevo ecosistema medicati.gl/RLYKwP

Cebrián Herreros, M. (2004). La información en televisión. Barcelona: Gedisa.

Freire, P. (1989). La educación como práctica de la libertad. Madrid: Siglo XXI.

Freire, P. (1992). Pedagogía del oprimido. Madrid: Siglo XXI.

Giroux, H. (1990). Los profesores como intelectuales. Barcelona: Piadós.

Haye, R. (1995). Hacia una nueva radio. Argentina: Paidós.

Haye, R. (1998). El impacto cultural de la radio. Recuperado de http://uncoma.academia.edu/RicardoHaye/Papers/419662/El Impacto Cultural De La Ra dio

Kaplún, M. (1976). La producción de programas radiofónicos. Quito: CIESPAL.

Kaplún, M (1997). La Eudocominicación. De medios y fines en comunicación. Revista Latinoamericana de Comunicación Chasqui, 58. Recuperado de http://chasqui.comunica.org/kaplun.htm.

Lalinde, A. M. (1992). La noticia: construcción de la realidad, en Industrias culturales, comunicación, identidad e integración latinoamericana. México: Ed. Opción.

Martín- Barbero, J (2002). La educación desde la comunicación. Eduteka Tecnologías de Información y Comunicaciones para Enseñanza Básica y Media. Recuperado de http://www.eduteka.org/pdfdir/SaberNarrar.pdf.

Martin- Barbero, J (2005). Los oficios del comunicador. Revista Co-herencia, 115-143. Recuperado de ttp://redalyc.uaemex.mx/pdf/774/77420206.pdf.

Martín-Barbero, J (1990). Teoría-investigación-producción en la enseñanza de la comunicación. Diálogos de la Comunicación, FELAFACS.

Martin-Barbero, J. (2010). La Universidad vive hoy en el mundo una relación esquizofrénica con la sociedad. XIII Encuentro de FELAFACS. La Habana. Recuperado de http://www.cubadebate.cu/.

Mata, M. C. (2012). La Radio: Una relación comunicativa. Revista latinoamericana de las Facultades de Comunicación social. Diálogos de la comunicación. Recuperado de http://conociendolaradio.bligoo.com.co/media/users/13/653504/files/72543/La Ra dio Una Relaci $n$ Comunicativa.pdf

Mata, M. C. (1998). Saber sobre la radio. Signo y Pensamiento, 33. 
Costales Pérez, Z.; Badía Valdés, A. T. Estrategias para la formación profesional en periodismo radiofónico: experiencias y propuestas

Mondragón, H. (1998). De la ansiedad al método pedagógico en los docentes universitarios. Seminario de Capacitación docentes UAO. Universidad Javeriana.

Morley, D. (1996). Televisión, audiencias y estudios culturales. Buenos Aires: Editorial Amorrortu editores.

Prieto, D. (2006). El interparendizaje como clave de la educomunicación. Mediaciones, 6.

Radio Rebelde. (2018). La radio: sonido para ver. Recuperado de http://www.cmhw.cu/radio-adentro/11749-la-radio-sonido-para-ver

UNESCO. (2012). Informe de Seguimiento de la EPT en el Mundo. Los jóvenes y las competencias: Trabajar con la educación. Paris: Ediciones UNESCO.

\section{AUTORAS}

\section{Zenaida Costales Pérez}

Periodista y Profesora Titular de la Facultad de Comunicación de la Universidad de La Habana. Correo electrónico costaleszenaida@gmail.com

Http://0000-0001-9561-5544

\section{Ana Teresa Badía Valdés.}

Profesora Titular de la Facultad de Comunicación de la Universidad de La Habana. Correo electrónico abadia@fcom.uh.cu

http://000-0003-2431-9684 